\title{
Comparative Observation of the Action Potential of Autonomic Nerve with E.E.G.
}

\author{
By \\ Osamu Orihara \\ (折 原 修)
}

(From the Surgical Clinic of Prof. S.-T. Katsura, Faculty of Medicine, Tohoku University, Sendai)

(Received for publication, June 17, 1952)

Correlation between the brain and the autonomic nerves have been studied by many authors from anatomical as well as physiological standpoints, but still many there are left to be clarified.

A great number of articles of E.E.G. have been published after the discovery of E.E.G. by Berger, and contributed much both to the brain physiology and clinical medicine. On the other hand, enormous advance in our understanding of the action potentials of peripheral nerves was established by many investigators following the brilliant works by Erlanger \& Gasser. Standing on such a ground of recent advance in neurophysiology; correlations of brain and peripheral nerves were studied by some authors, that is, studies of brain and cord by Davis etc., 1),2),3),4) those of brain and peripheral nerves by Gibbs, ${ }^{5)}$ Gellhorn, ${ }^{6)}$ and those concerning brain and autonomic nerves including vagus by Bailey \& Bremer, ") Darrow etc. ${ }^{81}$

As I studied the action potentials of brain, (E.E.G.) as related to those of autonomic nerves, some results obtained thereby I shall report here.

\section{EXPERIMENTAL \\ Method}

34 rabbits and cats were employed, all were anesthetized with urethane ( $1 \mathrm{~g}$. per kg. subcutaneously). N. sympathicus and vagal nerve were both severed and thoroughly separated about $3 \mathrm{~cm}$. long from the surrounding soft tissues and each was put on a pair of electrodes. In such procedures I took special care not to pull the nerves strongly. E.E.G. was led from frontal part of the skull of both sides with needle electrodes, common earth electrode being placed on the ear of the same side of the severed nerves. Action potentials of the brain and the autonomic nerves were led to amplifiers for E.E.G., specially constructed by Assist. Prof. 
Watanabe and his collaborator Matsuo of the Department of Electrical Engineering of Tohoku University. The amplified potential changes were recorded photographically, and yet all simultaneously. Hypothalamic nuclei were located accurately by Hosley-Clark's apparatus and a small area (about $1 \mathrm{~mm}$. in diameter) was electrocoagulated, the affected area was always confirmed afterwards by histological examination.

\section{Results}

\section{Changes of action potential of cervical autonomic nerves and E.E.G. due to some drugs}

1) About the judgement of records of action potential

Histogram of frequency of action potential waves of nerves and E.E.G. was constructed. The abscissa is scaled by logarithms of 39.2, 24, 15.5, 9.8, 6.2, 3.9, 2.4 per sec., and the ordinate is per cent of frequency of appearance among totals. Such histograms of E.E.G. and action potentials of autonomic nerves were compared each other before and after giving some drugs or stimulations of nerves. The changes amounting over than three times of mean error of histogram was decided as significant; in addition to this, changes in amplitude were also taken into consideration for the final judgement as a whole.

2) Spontaneous action potential of autonomic nerve at neck (Figs. 1, 2. Table I)

Amplitude of spontaneous action potential of sympathetic nerve is several to $15 \mu \mathrm{V}$., and its histogram of frequency is shown in Fig. 1. The
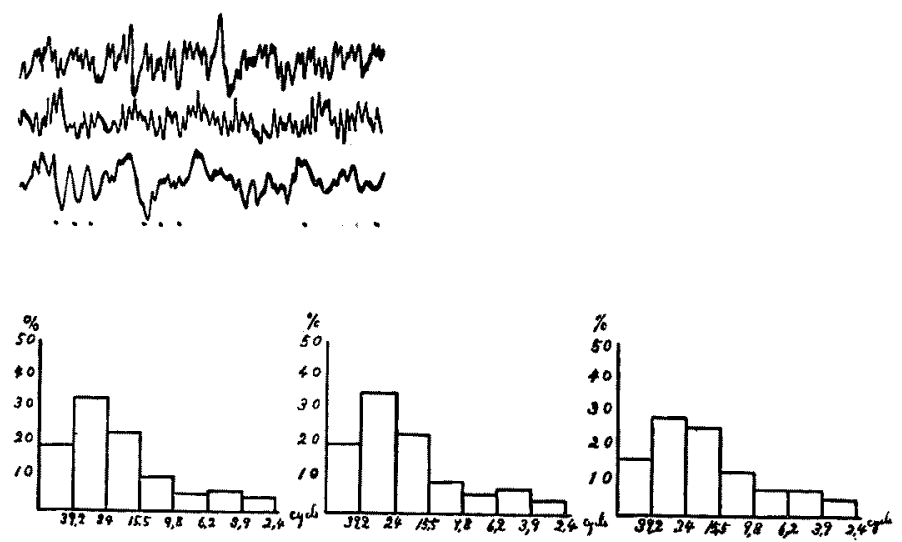

Fig. 1. Spontaneous action potential and its histogram of frequency. From the top downward action potential of sympathetic nerve, vagal nerve and E.E.G. Time: $1 / 100$ second. 


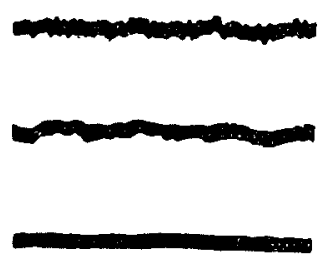

Fig. 2. Action potentials of the sympathetic (upper) and vagal nerve (middle) and E.E.G. (lower) when short-circuit is established (see explanation in text).

\section{TABLE I}

Frequency of Occurrence (in \% of total number) of Action Potentials of Various Periods (expressed in cycles per second)

\begin{tabular}{c|rl|rr|rr|}
\hline Cycle per second & \multicolumn{2}{|c|}{ Sympathetic nerve } & \multicolumn{2}{|c|}{ Vagal nerve } & \multicolumn{2}{|c}{ E.E.G. } \\
\cline { 1 - 7 } 39.2 & 18.9 & 3.1 & 20.4 & 2.4 & 16.6 & 2.7 \\
24.0 & 33.6 & 2.2 & 35.0 & 1.5 & 28.7 & 1.9 \\
15.5 & 23.2 & 2.5 & 23.3 & 1.2 & 26.3 & 1.3 \\
9.8 & 9.9 & 1.2 & 9.1 & 1.1 & 13.1 & 1.0 \\
6.2 & 5.6 & 0.6 & 5.7 & 0.9 & 7.4 & 0.8 \\
3.9 & 6.5 & 0.8 & 6.5 & 1.0 & 7.6 & 0.9 \\
2.4 & 4.8 & 1.1 & 4.1 & 0.7 & 5.2 & 0.5
\end{tabular}

peak of greatest occurrence is found between 39.2 per sec. and 24 per sec. In Table I, will be found arithmetical mean and mean error of each part of this histogram of frequency. An example of action potential led from vagal nerve is shown in Fig. 1, its amplitude reaches several to $15 \mu \mathrm{V}$. and the peak of histogram of frequency is between 39.2 per sec. and 24 per sec. This potential change disappears (as shown in Fig. 2) when a pair of electrodes applied to nerve short-circuited, so that the potential change led from the nerve is obviously the potential change of nerve itself.

3) Potential change to adrenalin (Fig. 3)

5 rabbits were employed. $0.025 \mathrm{mg}$. of adrenalin pro $\mathrm{kg}$. in $1 \mathrm{cc}$. of $0.9 \%$ of natrium chloride solution was injected in an ear-vein and in a few minutes potential changes were photographed. The wave, as in Fig. 3 was observed; though its amplitude was not changed evidently, its frequency decreased in the fast wave from 39.2 to 24 per sec. in all cases. Changes of potential of vagal nerve were not constant. In E.E.G. fast wave (24-15.5 per sec.) decreased in all cases and also intermediate wave (9.8-6.3 per sec.), increased, however, slow wave (3.9-2.4 per sec.) in 3 cases. 

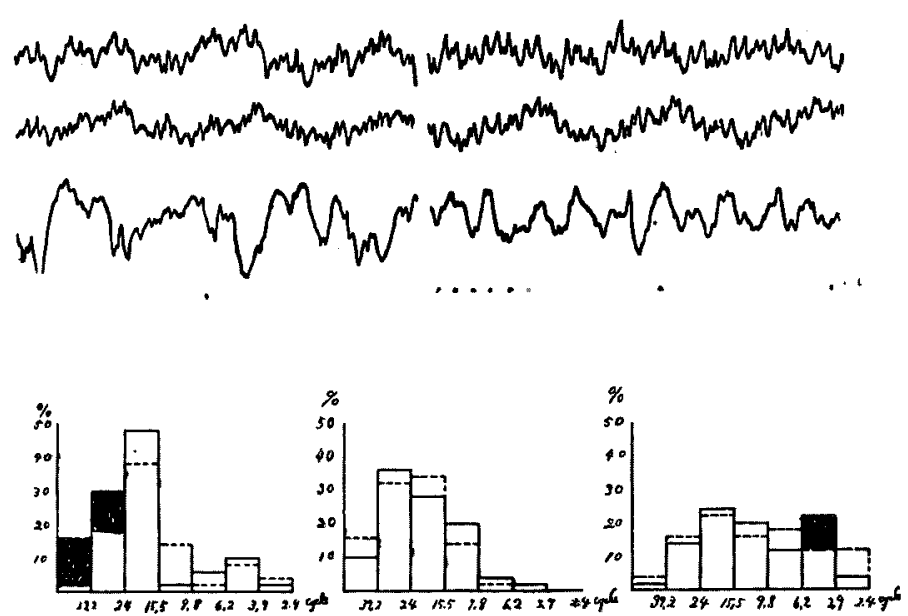

Fig. 3. Potential change due to adrenalin injection and histogram of frequency. From the top potential change of sympathetic nerve, vagal nerve and E.E.G. The left; before adrenalin injection. The right; after adrenalin injection. Histogram after adrenalin injection is shown with dotted line.

4) Potential changes to acetylcholine and metrazol (Fig. 4 \& 5)
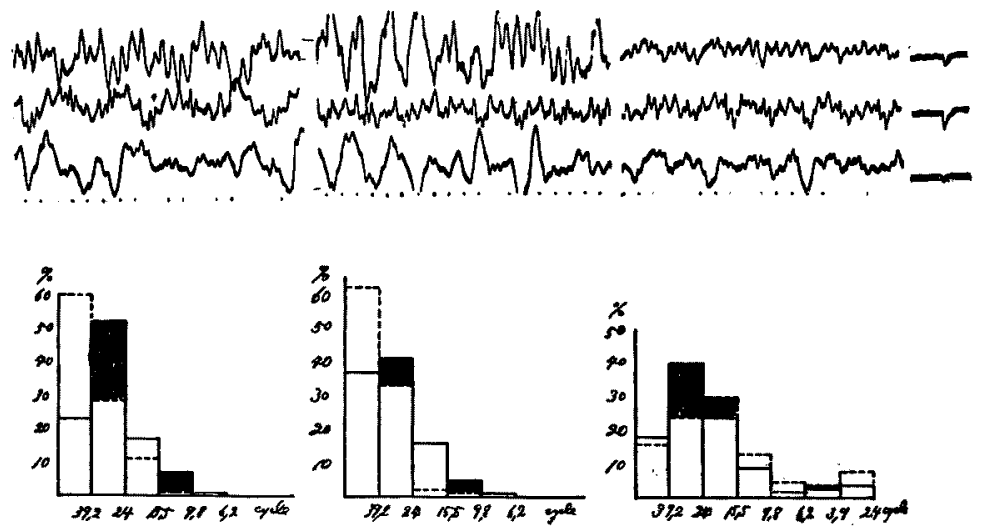

Fig. 4. Potential change to acetylcholine injection. From the left, before injection, acetylcholine injection and T.E.A.B. injection, From the top, potential change of sympathetic nerve, vagal nerve and E.E.G. Histogram after acetylcholine injection is shown with dotted line.

$0.05 \mathrm{mg}$. of acetylcholine or $0.1 \mathrm{cc}$. of metrazol in $1 \mathrm{cc}$. of $0.9 \%$ of natrium chloride solution were injected in an ear-vein in 4 cases and in a few minutes the potential changes were photographed. Convulsion did not occur. Amplitude of potential of both nerves turns into twice 


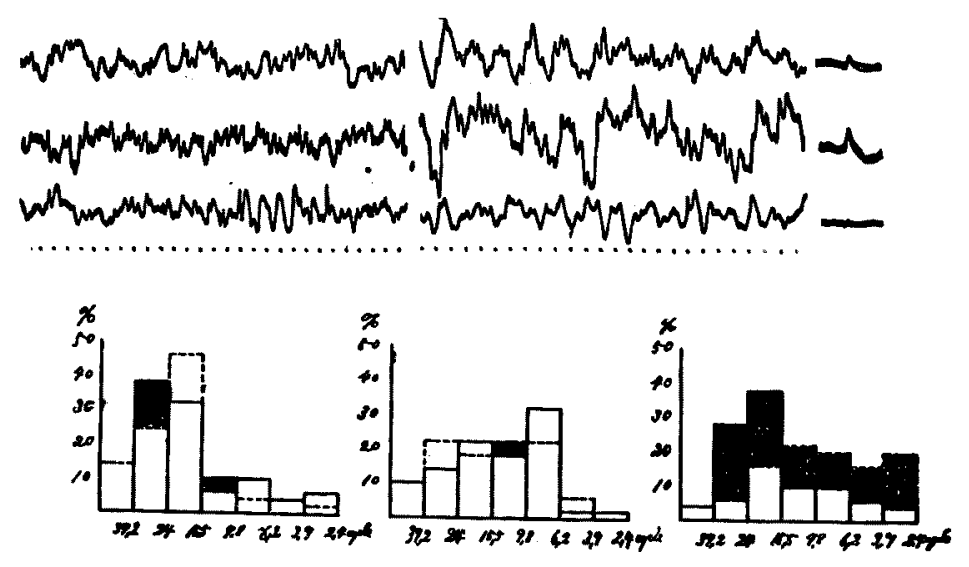

Fig. 5. Potential change due to metrazol injection. From the top, potential change of sympathetic nerve, vagal nerve and E.E.G. Histogram after metrazol injection is shown with dotted line.

the control size. Fast wave (39.2-24 per sec.) of sympathetic nerve decreased and intermediate wave (15.5-9.8 per sec.) increased in 3 cases. In the vagal nerve, fast wave (39.2-24 per sec.) and intermediate wave (15.5-9.8 per sec.) decreased in 3 cases, in E.E.G. fast wave (39.2-24 per sec.) decreased, intermediate wave (24-15.5 per sec.) and slow wave (6.23.9 per sec.) increased in 3 cases.

Due to metrazol, change of frequency of sympathetic nerve was as follows; fast wave (39.2-24 per sec.) decreased in 3 cases and intermediate wave (15.5-9.8 per sec.) in all. In vagal nerve intermediate wave (15.59.8 per sec.) increased in 3 cases, fast wave (39.2-15.5 per sec.) of E.E.G. decreased in all cases, and intermediate wave (15.5-9.8 per sec.) in 3 cases, intermediate wave of 9.8-6.2 per sec. and slow wave (6.2-2.4 per sec.) increased in all cases. When $40 \mathrm{mg}$. of T.E.A.B. was injected into a vein, its amplitude decreased markedly.

5) Potential change due to pilocarpine (Fig. 6)

When $1 \mathrm{mg}$. pro $\mathrm{kg}$. of pilocarpine in $0.9 \%$ of natrium chloride solution was injected into vein, amplitude usually increased markedly and especially in E.E.G. Change of frequency of sympathetic nerve potential was not constant, but intermediate wave (15.5-9.8 per sec.) of vagal nerve and also in E.E.G. increased in all cases. When $1 \mathrm{mg}$. of atropine pro $\mathrm{kg}$. was given intraveneously at this time, the change due to pilocarpine disappeared instantaneously, and the potential returned to the state before pilocarpine-injection.

Results in this section above may be summarized as follows.

1. In action potential of sympathetic nerve, intermediate wave 

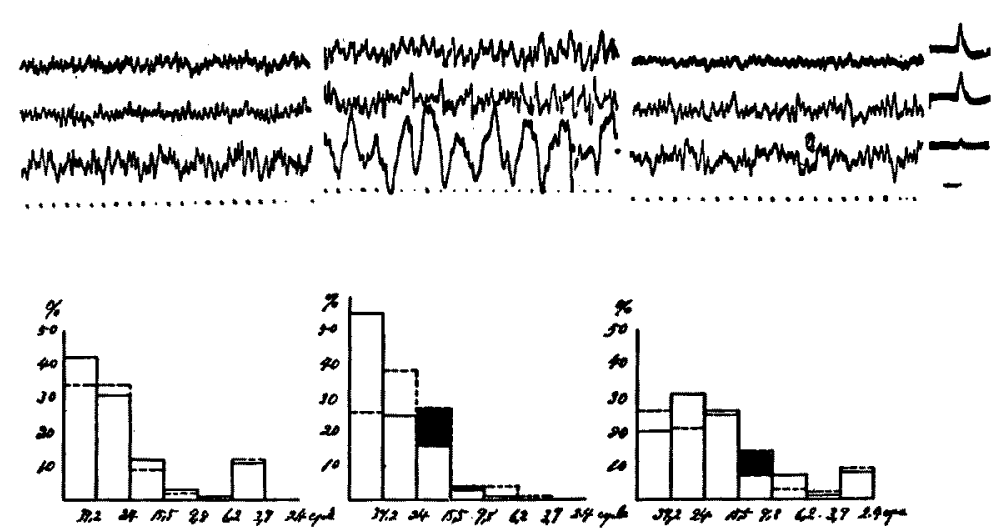

Fig. 6. Potetial change due to pilocarpine and atropine injection. From the left, before injection, pilocarpine injection and atropine injection. From the top, potential change of sympathetic nerve, vagal nerve and E.E.G. Histogram after pilocarpine injection is shown with dotted line.

$(10-15 \mu \mathrm{V}$.) and fast wave (several $\mu \mathrm{V}$.) were superimposed on slow wave $(20-30 \mu \mathrm{V}$.)

2. Potential changes of sympathetic nerve to adrenalin, metrazol and acetylcholine, that stimulate sympathetic nerve, are divided into two sorts. The one: fast wave (39.2-24 per sec.) decreases and there is no evident change in amplitude, in E.E.G. at the same time slow wave (6.2-3.9 per sec.) decreases. Such a change is observed to adrenalin injection. The other: fast wave (39.2-24 per sec.) and intermediate wave (15.5-9.8 per sec.) decrease. With markedly increased amplitude and in E.E.G. slow wave (6.2-3.9 per sec.) increases with high potential. Such a change is observed at acetylcholine and metrazol injection.

3. Changes in action potential of vagal nerve due to pilocarpine are an increase in amplitude and increase of intermediate wave (15.59.8 per sec.); in E.E.G. there are similar changes.

\section{Potential change of autonomic nerves and E.E.G. when hypothalamic nuclei were electrocoagulated}

Cats were used being anesthetized with urethane ( 1 g. per kg.), and potential change of autonomic nerve and E.E.G. were led as above described. To destroy hypothalamic nuclei an electrode was inserted in hypothalamus by means of Hosley-Clark's apparatus, and electrocoagulation (Phase of $1 \mathrm{~mm}$. in diameter) was performed. After the experiment had been finished, the brain was removed, fixed and stained for accurate identification of the destroyed parts.

1) Potential change of autonomic nerves and E.E.G. when medial 

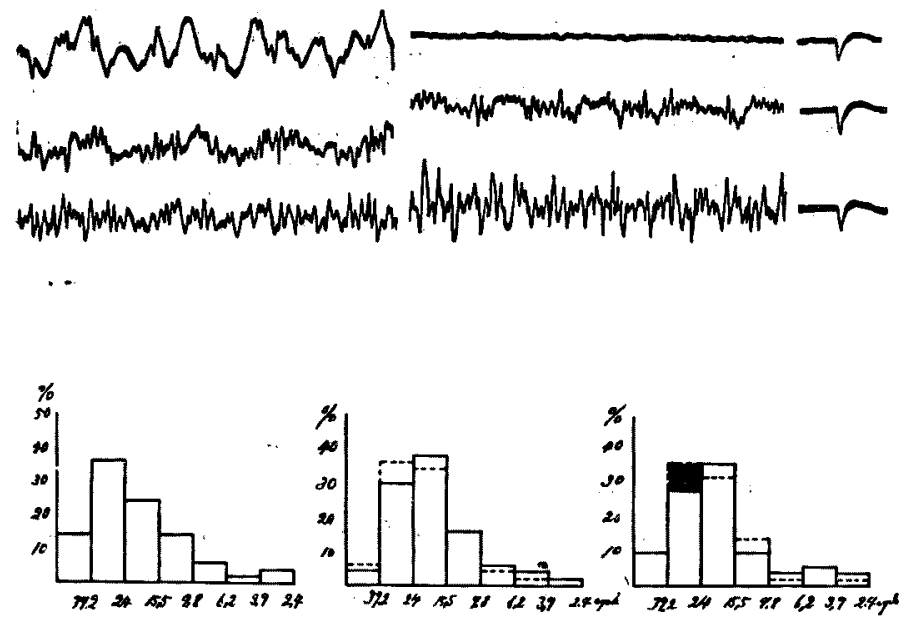

Fig. 7. Potential change due to electrical coagulation of anterior medial part of n. opticus. From the left, before coagulation and after coagulation. From the top, action potential of sympathetic nerve, vagal nerve and E.E.G. Histogram after coagulation is shown with dotted line.
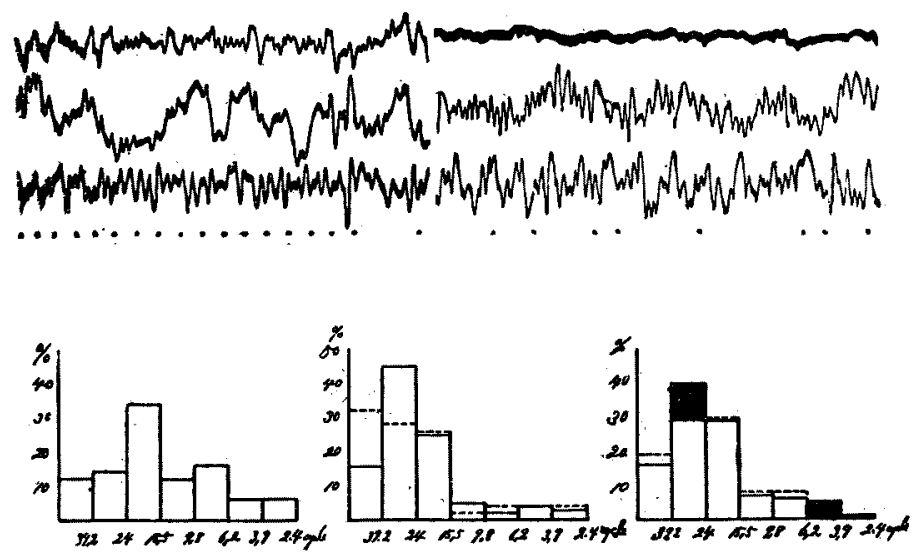

Fig. 8. Potential change due to electrical coagulation of nucl. paraventricularis. From the left, before coagulation and after coagulation. From the top, potential change of sympathetic nerve, vagal nerve and E.E.G. Histogram after coagulation is shown with dotted line.

anterior nuclei of n. opticus were destroyed (Fig. $7 \&$ 8)

Potential change of autonomic nerves following the electrocoagulation was more evident homolaterally in sympathetic nerve than in vagal nerve. As shown in Fig. 7, potential change was an almost complete disappearance of action potential in sympathetic nerve, and an increase 
of fast wave (39.2-24 per sec.) in vagal nerve, its amplitudes, however, being not enlarged. In E.E.G. fast wave (39.2-24 per sec.) increased when medial autonomic nuclei of 'n. opticus was electrocoagulated, and decreased fast wave (39.2-24 per sec.) when nucleus paraventricularis was electrocoagulated with enlarged potential $(30 \mu \mathrm{V}$.).

2) Potential change when nucl. supraopticus dorsalis and nucl. hypothalamicus dorsalis magnocellaris were electrocoagulated (Fig. 9)
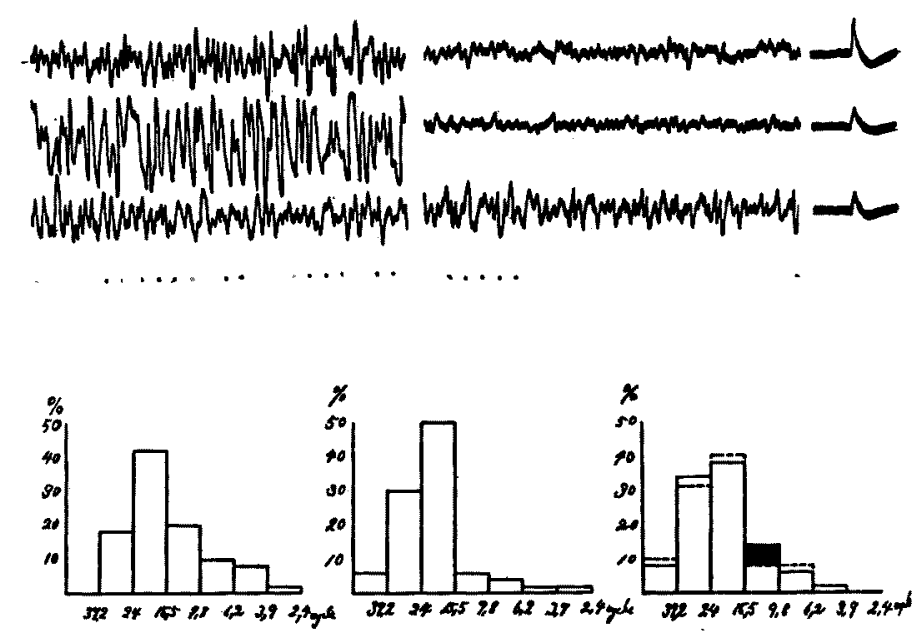

Fig. 9. Potential change due to electrical coagulation of nucl. supraopticus dorsolateralis and nucl. hypothalamicus dorsalis magnocellaris. From the left, before coagulation and after coagulation. From the top, potential change of sympathetic nerve, vagal nerve and E.E.G. Histogram after coagulation is shown with dotted line.

In the electrocoagulated side fast wave (39.2 per sec.) of sympathetic nerve increased without change in amplitude, and in vagal nerve the amplitude of action potential markedly decreased (to $10 \mu \mathrm{V}$. or less), fast wave (39.2 per sec.) increased, intermediate wave, however, (9.86.2 per sec.) decreased. In E.E.G. fast wave (24-15.5 per sec.) decreased and intermediate wave $(9.8-6.2$ per sec.) increased without any potential change:

3) Potential change when nucl. supraopticus dorsolateralis was electrocoagulated (Fig. 10)

In the electrocoagulated side potential of sympathetic nerve decreased, vagal nerve potential decreased also so markedly (to less than several $\mu \mathrm{V}$.) that its frequency became hardly measurable. In E.E.G. intermediate wave (15.5-9.8 per sec.) decreased without any potential change: 

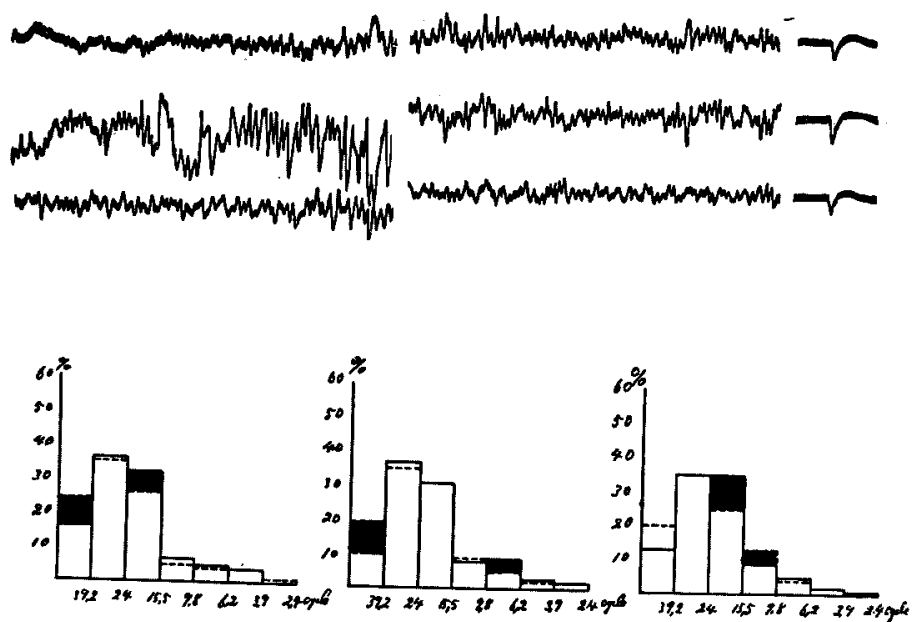

Fig. 10. Potential change due to electrical coagulation of nucl. supraopticus dorsolateralis. From the left, before coagulation and after coagulation. From the top, potential change of sympathetic nerve, vagal nerve, and E.E.G. Histolgram after coagulation is shown with dotted line.

\section{Summary:}

1. When medial anterior nuclei of $n$. opticus or nucl. paraventricularis were electrocoagulated, action potentials of sympathetic nerve disappeared in the coagulated side. In E.E.G. fast wave increased with increase in amplitude when medial anterior nuclei of $n$. opticus were electrocoagulated, and fast wave decreased, slow wave (6.2-3.9 per sec.) increased when nucl. paraventricularis were electrocoagulated.

2. When nucl supraopticus dorsalis and nucl. hypothalamicus dorsalis magnocellaris of the other side were electrocoagulated, action potential of vagal nerve got decreased especially.

3. When nucl. supraopticus dorsolateralis was electrocoagulated, action potentials on autonomic nerves became markedly reduced and in E.E.G. intermediate wave increased without any potential change.

III. Potential change of cut autonomic nerves and E.E.G. when autonomic nerves were cut at neck (Fig. 11)

Autonomic nerves were cut in one side, and at both cut ends were led its action potentials along with E.E.G. At the central stump the amplitude of potential of both the sympathetic and vagal nerve got decreased. In the sympathetic nerve, the fast wave (39.2-24 per sec.) decreased, and the intermediate wave (9.8-6.2 per sec.) increased, while in the vagal nerve fast wave (24-15.5 per sec.) decreased, and the intermediate wave 

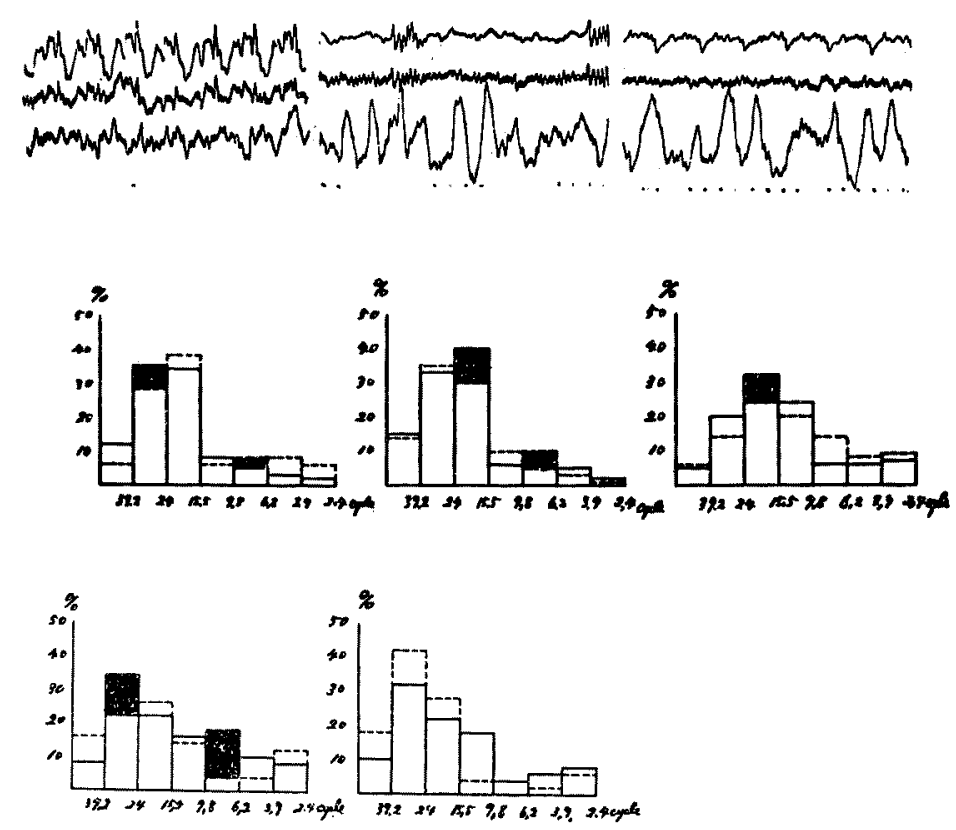

Fig. 11. Potential change at cut end of autonomic nerves. From the left, before cuting, after cuting at central end and at peripheral end. From the top, potential change of sympathetic nerve, vagal nerve and E.E.G. Histogram after cuting of nerves is shown with dotted line, the top shown the central cut end and the bottum the peripheral cut end.

(9.8-6.2 per sec.) increased. In E.E.G. the potential increased markedly. At the distal cut end the amplitude decreased evidently both in the sympathetic and vagus nerves. As to its frequency the fast wave (39.2-24 per sec.) of sympathetic nerve decreased, and the intermediate wave (9.86.2 per sec.) increased in all instances, but no constant potential changes were observed in the vagal nerve.

In short, when autonomic nerves were cut and potential changes were led at both cut ends, amplitudes of potential of sympathetic nerve as well as frequency of the fast wave decreased and frequency of the intermediate wave increased.

\section{Discussion}

Action potentials of peripheral nerves were studied by Erlanger, Gasser, Bishop and Adrian, ${ }^{10}$ ) followed by Detlev \& Frank, ${ }^{11}$ ) Tasaki \& Fujita $^{12)}$ Bullock ${ }^{13)}$ and Young. ${ }^{14)}$ These authors all investigated chiefly of spike potentials of nerves. With E.E.G. apparatus we observed the waves of 39.2-24 per sec. in the autonomic nerves of cervical region. The 
action potentials of such a whole nerve trunk were not studied so far. Such potential changes as I observed were definitely those of the autonomic nerves themselves, because the potentials disappeared when two electrodes were shortcircuited, and they changed characteristically due to some drugs known especially to affect the autonomic nervous activity.

Cortical E.E.G. which is led from frontal area of rabbit was studied by Ito \& Kitamura. They said that E.E.G. does not change particularly by location. I led E.E.G. from frontal area that was said to be related to the autonomic nerve. While Davis says that E.E.G. of rabbit has a frequency of 13.5-8 per sec., in our experiment fast waves were observed more abundantly in 2 cases of rabbits anesthetized by urethane than in normal animals. Such finding may be ascribed to effect of urethane injection.

Fast wave (24-15.5 per sec.) in sympathetic nerve decreased by adrenalin injection and in vagal nerve there was observed no particular change; in E.E.G. slow wave of 6.2-3.9 per sec. increased with slightly diminished amplitude. Davis ${ }^{11}$ says that $\alpha$ and $\beta$ waves increase with increased amplitude due to sensory stimulation. Bartiley \& Hembecker ${ }^{15}$, reported the amplitude diminished in some conditions, and Lennox \& Gibbs $^{51}$ say that the influence of adrenalin to E.E.G. is insignificant. I consider that adrenalin plays influences upon E.E.G. through a reflex from its periphery, because the potential change of autonomic nerves was observed at both cut ends at the neck.

In single fibre preparations spike potentials were observed due to acetylcholine (Bullock, ${ }^{13)}$ Young ${ }^{14)}$ ). The action potentials of autonomic nerve trunk at neck were different from it and rather large potential waves were seen in the sympathetic nerve with a decrease of fast waves and the intermediate wave. In the vagal nerve, the change in frequency was the same but its amplitude was not changed. The change of E.E.G. due to acetylcholine has been discussed as "acetylcholine discharge" by many authors, Miller, Stavraky \& Woonton, ${ }^{16)}$ Foerster, ${ }^{17)}$ Bonnet \& Bremer ${ }^{18)}$ Chatfied Dempsy. ${ }^{19)}$ My results were almost the same as these authors', that is, slow waves of high potential and fast wave of 2415.5 per sec, were increased.

Change of action potential of the sympathetic nerve due to metrazol was almost the same as due to acetylcholine injection, but its amplitude was less than the amplitude in cases of acetylcholine injection. In vagal nerve the intermediate wave increased with enlarged amplitude and in E.E.G. the intermediate wave and the slow wave increased. That is, potential change of sympathetic nerve and E.E.G. due to adrenalin, acetylcholine and metrazol (all these drugs stimulate the sympathetic nerve), is divided into two sorts, one is such that in the sympathetic nerve 
fast wave and slow wave of E.E.G. decreases, another is such that the fast wave and the intermediate wave decreases in sympathetic nerve and slow wave of E.E.G. increases.

Potential change of sympathetic nerve to pilocarpine is not significant and in vagal nerve fast wave increases, in E.E.G. intermediate wave and slow wave of high potential increase markedly. The same result was reported by Darrow \& Pathman, ${ }^{10)}$ Bailey \& Bremer, ${ }^{7}$ Darrow, Green, Davis \& Garol. ${ }^{8)}$ When vagal nerve is stimulated, its action potential of the intermediate wave increases and the same change is observed in E.E.G. Thus metrazol may lead to stimulation of the vagal nerve because these changes are observed also when metrazol is given.

Gerard, Marshall \& Saul,21) Kornmüller ${ }^{22)}$ and Kennard ${ }^{23)}$ reported of the correlation between hypothalamus and E.E.G.: the former authors say that the hypothalamus has no relation to E.E.G. and the latter authors say that the hypothalamus has some influences on the cortical E.E.G. We observed some changes in frequency and amplitude when one of these nuclei, that is, medial anterior nucl. of $n$. opticus, nucl. supraopticus dorsolateralis, nucl. hypothalamicus dorsalis magnocellaris and nucl. paraventricularis, was electrocoagulated. These nuclei show influences on cortical E.E.G. Moreover, medial anterior nucl. of n. opticus, nucl. paraventricularis and nucl. hypothalamicus dorsalis magnocellaris are closely connected with action potential of the cervical sympathetic nerve, and nucl. supraopticus dorsolateralis with action potential of both sympathetic and vagal nerves at neck.

\section{GoncLusion}

In concluding my report I would emphasize the following facts:

1. Action potential of sympathetic and vagal nerve at neck is 39.22.4 per sec. in freqnecy, the majority being $39.2-15.5$ per sec.

2. Change of action potential of sympathetic nerve at neck due to adrenalin is a decrease in fast wave (39.2-15.5 per sec.) and in E.E.G. a decrease in slow wave (6.2-3.9 per sec.).

3. Of the action potentials of sympathetic nerve at neck the fast wave (39.2-24 per sec.) and the intermediate wave (15.5-9.8 per sec.) decrease and in E.E.G. the slow wave (6.2-3.9 per sec.) increases due to acetylcholine. As to the action potential of sympathetic nerve to metrazol the intermediate wave (15.5-9.8 per sec.) and slow wave (6.2-3.9 per sec.) decrease, in the vagal nerve the intermediate wave (15.5-9.8 per sec.) and in E.E.G. the intermediate wave (15.5-9.8 per sec.) and slow wave 6.2-3.9 per sec.) increase.

4. Concerning the action potential of vagal nerve at neck, pilocarpine increases the intermediate wave (15.5-9.8 per sec.). In E.E.G. 
the intermediate wave (15.5-9.8 per sec.) with high potential increases in frequency.

5. Damage of medial anterior nucl. of n. opticus, nucl. paraventricularis, nucl. supraopticus dorsalis and nucl. hypothalamicus dorsalis magnocellaris affects the cortical E.E.G., lesion of the former two results in a disappearance or marked decrease of action potential of the sympathetic nerve, lesion of the latter two results in changes in action potentials of sympathetic and vagal nerve.

6. Potential changes are observed at both cut ends of both the sympathetic and the vagal nerve when they are cut at the neck.

I express my appreciation to Prof. S. -T. Katsura and Prof. K. Motokawa whose cordial guidance made me carry out the present investigation, and to Prof. K. Matsuda for his assistance in Preparation of this manuscript.

\section{References}

1) Davis, Ann. Rev. Physiol., 1939, 1, 345.

2) Bonnet and Bremer, Compt. rend. soc. biol., 1937, 126, 1271.

3) Libet and Gerard, Amer. J. Physiol., 1938, 123, 128.

4) Miller, Stavraky and Woonton, Amer. J. Physiol., 1938, 123, 147.

5) Gibbs and Lennox, Arch. Inn. Med., 1937, 60, 154.

6) Gellhorn, J. Neurophysiol., 1947, 10, 125.

7) Bailey and Bremer, J. Neurophysiol., 1938, 1, 405.

8) Darrow, Green, Davis and Garol, J. Neurophysiol., 1944, 7, 217.

9) Motokawa, Noha, Tokyo, 1947.

10) Höber, Lehrbuch der Physiologie, Bern, 1939.

11) Detlev and Frank, Ann. Rev. Physiol., 1938, 1, 385.

12) Tasaki and Fujita, J. Neurophysiol., 1948, 11, 311.

13) Bullock, J. Neurophysiol., 1948, 11, 343.

14) Young, Philos. Trans., 1939, 229, 465.

15) Bartley and Hembecker, Amer. J. Physiol., 1938, 121, 21.

16) Miller, Stavraky and Woonton, J. Neurophysiol., 1940, 3, 131.

17) Foerster, Arch. Neurol. \& Psychiatr., 1945, 54, 391.

18) Bonnet and Bremer, Comp. rend. soc. biol., 1937, 126, 1271.

19) Chatfied and Dempsy, Amer. J. Physiol., 1942, 135, 633.

20) Darrow and Pathman, Fed. Proc., 1943, 2, 9.

21) Gerard, Marshall and Saul, Proc. Soc. Exp. Biol., 1933, 301, 1123.

22) Jung and Kornmüller, Arch. Psychiatr. Nervenkr., 1938, 109, 1.

23) Kennard, J. Neurophysiol., 1943, 1, 233. 